\title{
Tilted letters and tilted words: A possible role for principal axes in visual word recognition
}

\author{
JON DRIVER \\ University of Cambridge, Cambridge, England \\ and \\ GORDON C. BAYLIS \\ University of South Carolina, Columbia, South Carolina
}

\begin{abstract}
Subjects made lexical decisions to columnar letter strings in which every letter was either upright or tilted $90^{\circ}$ clockwise as if the whole letter string had been rotated from the horizontal. Lexical decisions were faster in the latter case. The advantage for the tilted format was also found when all strings were presented in aLtErNaTiNg CaSe or in uppercase, so this advantage cannot be due to preservation of the tilted words' global shape. The cost for the upright-letter format increased with the number of letters in the columnar strings. These data suggest that word recognition may involve shape description or position coding relative to a reference frame based on the principal axis of the letter string.
\end{abstract}

Many authors have suggested that the whole is more than the sum of its parts in word perception. Certainly, a wide range of evidence suggests that recognizing a word does not solely consist of identifying the constituent letters as if each were presented in isolation. Instead, individual letter perception can benefit from the structure of the word as a whole. For example, the well-known wordsuperiority effect demonstrates that compared with presentation alone or in an unpronounceable string, identification of a letter can improve if it is embedded within a familiar word or pronounceable string (e.g., Henderson, 1987; Huey, 1908/1968; McClelland \& Rumelhart, 1981; Reicher, 1969).

In addition to benefiting from top-down effects of this kind, which presumably accrue from familiarity, letter perception within words may also benefit from global structure in a purely bottom-up manner. The possible contribution of one such bottom-up factor provides the focus of the present paper. The global configuration of a letter string may provide a reference frame relative to which the constituent letters are described. This possibility arises from research into the perception of nonlinguistic visual shapes by people and machines. We describe this work briefly below, before considering its possible relevance to word perception.

Marr and Nishihara (1978) argued on computational grounds that shapes should be described relative to their

J.D. was supported by a grant from the Science and Engineering Research Council (U.K.). G.C.B. was supported by U.S. Office of Naval Research Contract N00014-91-J-1735. Our thanks to Rich Ivry for generous use of his lab space, and to Geoffrey Loftus, Robert Proctor, and an anonymous referee for helpful comments on an earlier draft. Correspondence concerning this article may be addressed to J. Driver, Department of Experimental Psychology, University of Cambridge, Downing Street, Cambridge CB2 3EB, England. intrinsic principal axes of elongation and/or symmetry. The resulting object-centered descriptions would be (relatively) independent of viewpoint, and thus help to solve the problem of recognizing the same shape from different views. Evidence that axis-based descriptions of this kind are formed during nonlinguistic shape perception has been provided by, among others, Humphreys and Palmer, both of whom took advantage of the fact that some shapes are unusual in having no single unambiguous axis of elongation or symmetry (e.g., squares are equally elongated and symmetrical about several axes). Humphreys (1983) found that subjects were slower to judge that two squares had the same shape when one was rotated $45^{\circ}$ in the picture-plane than they were to judge that two isosceles triangles had the same shape across the same transformation. He suggested that subjects could readily derive axis-based representations for the isosceles triangles because of their unambiguous principal axis. These objectcentered representations would match across viewpoint changes, such as the $45^{\circ}$ rotation, and hence facilitate the matching of shape. In contrast to isosceles triangles, squares have no single principal axis. In such ambiguous cases, a preference is known to emerge for gravitationally vertical axes (Rock, 1973). Thus, the upright squares are likely to be described relative to a vertical axis running centrally and parallel to their sides. By contrast, the $45^{\circ}$ rotated squares would be described relative to a vertical axis which now runs along their diagonal. This would lead to an object-centered description that is different from that for the upright square (i.e., it is now described as a diamond; see Mach 1914/1959), hence producing the difficulty in matching across rotations for squares (see also Humphreys \& Quinlan, 1988).

Palmer and colleagues (e.g., Palmer, 1980, 1985) have provided additional evidence for axis-based descriptions in nonlinguistic shape perception which shows that the 
perception of local structures can depend on axes determined by the global configuration. For example, subjects required to judge whether a central shape is a square or a diamond are influenced by whether surrounding shapes are aligned with the diagonal axis of the central shape or with one of its parallel axes (the former alignment leading to a diamond interpretation, and the latter to an interpretation of the very same central shape as a square). Similar factors resolve the ambiguous direction of "pointing" that is perceived for equilateral triangles. These shapes are seen to point along the axis of symmetry of the global configuration (e.g., Attneave, 1968; Palmer \& Bucher, 1981). Such data imply that the visual system often describes the component shapes within a scene relative to a principal axis of elongation or symmetry determined by the configuration as a whole.

In this paper, we examine whether similar principles apply during the shape-perception processes involved in visual word recognition. If so, we would expect the recognition of words to vary according to the configurations in which their component letters are embedded. When presented individually, letters should generally be described relative to a vertical axis, since this is their principal axis of elongation (and also of symmetry for several letters). Moreover, as discussed earlier, there is a general preference for vertical reference frames when other factors are equal (e.g., Rock, 1973). On the other hand, given the conventional format for English text, letters in words should generally be described relative to a horizontal axis, since this is invariably the major axis of stimulus elongation.

Our experiments examine whether the global configuration of a letter string influences perception in a manner consistent with description of component shapes (letters) relative to the principal axis of the global configuration (the string). The task was lexical decision for columnar letter strings. There were two possible formats: The letters in the string were either all upright or all tilted $90^{\circ}$ clockwise, as if an entire horizontal letter string had been translated through $90^{\circ}$ (see Figure 1A for an example of each of these two formats). The rationale for this manipulation was as follows. In the upright-letters format, the component letters remain in their familiar orientation relative to the observer, but become misoriented relative to the principal axis of the column as a whole (which now runs vertically, rather than horizontally as in the conventional text format of rows). In the tilted format, conversely, the component letters are individually misoriented relative to the observer, but remain normally oriented with respect to the principal axis of the configuration.

If word perception involves letter-recognition processes that are unaffected by the global configuration of the string, recognition should be determined by the ease of identifying individual letters at the two orientations (i.e., tilted and upright). Although single-letter identification is not always affected by orientation (Corballis \& Nagourney, 1978; Corballis, Zbrodoff, Shetzer, \& Butler, 1978; Eley, 1982; Koriat \& Norman, 1989; White, 1980), any effect always takes the form of an advantage for upright

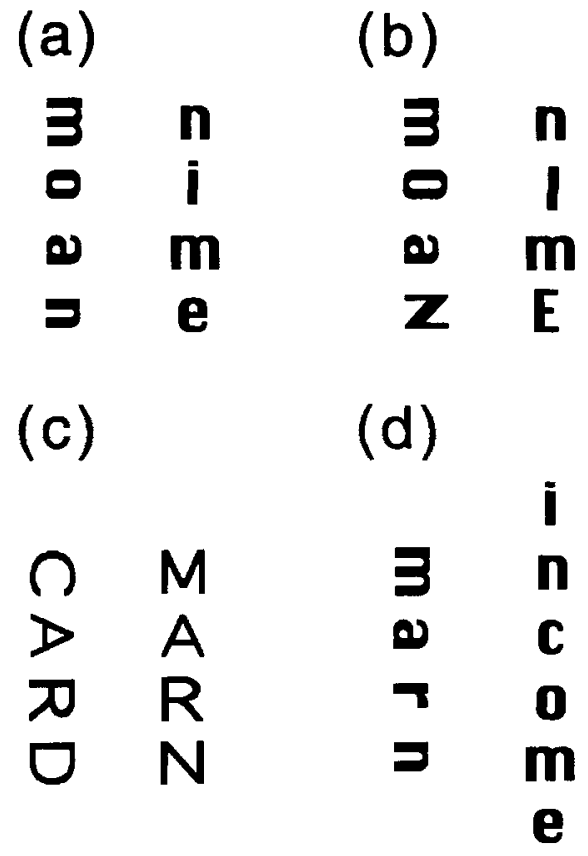

Figure 1. Examples of the two columnar stimulus formats presented for lexical decision in Experiments 1 through 4. Words and nonwords were equally likely to be presented in either format. (a) The left column illustrates a word in the tilted format, and the right col umn shows a nonword in the upright format used in Experiment 1. (b) Examples of stimuli for Experiment 2, which were just as for Experiment 1 , except that they were presented in alternating case. (c) Examples of uppercase stimuli from Experiment 3, with a tilted word illustrated on the left and an upright nonword shown on the right. (d) Examples of stimuli from Experiment 4, which had string length as an additional factor. A four-letter tilted nonword is illustrated on the left, and a six-letter upright word is shown on the right.

characters (e.g., Jolicoeur \& Landau, 1984). Thus, if word recognition relies on letter-perception processes that are immune to the global configuration, lexical decision should be as good in the upright condition as in the tilted condition, or better (i.e., faster). However, if letters are normally described relative to the principal axis of the string, lexical decisions should be faster, or more accurate, in the tilted case, since the letters retain their usual relation to the principal axis in this format, despite their misorientation relative to the subject. To test these opposing predictions, we compared lexical decision speed and accuracy for strings with the two columnar formats shown in Figure 1A, using two levels of frequency in the case of our word stimuli.

\section{EXPERIMENT 1}

\section{Method}

Subjects. The 30 subjects were lower-division psychology undergraduates at the University of California, San Diego, who received course credit for participation. All reported normal or corrected acuity.

Apparatus and Materials. The experiment was conducted on either a Zenith 286 or a Compaq $386 / 20$ microcomputer. The stimuli were presented in "stroked" font in graphics mode to enable 
accurate placement of stimuli within the display field. EGA doublepaged graphics mode was used to ensure that the onset or offset of stimuli occurred within a single frame. Displays were presented on an amber monochrome EGA monitor (Samsung). The subjects responded on the " $\mathrm{Z}$ " and "/" keys on the standard extended keyboard, depending on whether a word or a nonword was presented, and using the index finger of either hand. Half of the subjects responded to words with their preferred hand, and half responded with their nonpreferred hand.

The materials were taken from Besner and McCann (1987), and were all four-letter strings. They comprised 120 high-frequency words, 120 low-frequency words, and 240 pronounceable nonwords. Any effects of word frequency are largely tangential to our main concerns and were examined at this stage for information only, rather than to test a specific hypothesis. All the strings were presented in lowercase (Turbo Pascal sans-serif font), with each item appearing only once for every subject. Each letter was constructed within a square matrix that subtended $0.5^{\circ}$ vertically $\times 0.5^{\circ}$ horizontally at the viewing distance of $60 \mathrm{~cm}$. The letters in a string were placed $0.7^{\circ}$ apart vertically (center to center), to form a column centered at fixation.

Design. A within-subjects design was used, with two factors: format (upright vs. tilted letters; in both cases, vertical letter strings were presented-see Figure la for one example of each format); and string type (high frequency, low frequency, or nonword). These two factors were crossed to yield six conditions (high-frequency tilted, high-frequency upright, low-frequency tilted, low-frequency upright, nonword tilted, and nonword upright).

Procedure. The subjects had to make a lexical decision as quickly and accurately as possible by pressing one of two response keys under the index finger of either hand, the particular hands for positive and negative decisions being counterbalanced across subjects. They were shown examples of the two formats and were told that these formats were equiprobable and would come in an unpredictable sequence. A chinrest was used to control viewing distance, and it was stressed that the subjects should not tilt their heads. They were observed in order to ensure compliance with this instruction. Each trial began with a fixation cross presented for $500 \mathrm{msec}$ at the point where the letter string would be centered, followed by a fourletter string that remained until the subject responded. There was then a delay of $2 \mathrm{sec}$ before the sequence was repeated. The subjects were presented with eight blocks of 60 trials, the first block being discarded as practice. Half of the trials had the tilted format and half had the upright format, and these were randomly intermingled. The particular items that were chosen to appear in these formats varied randomly across subjects. A total of $25 \%$ of trials used high-frequency words, while $25 \%$ used low-frequency words and the remainder used nonwords. The six conditions appeared in the appropriate proportions within each block. Beyond these constraints, a different random sequence of trials was used for each subject.

At the end of each block, the subjects received feedback on their mean correct reaction time and their mean error rate. To ensure that the subjects were responding quickly but accurately, the computer displayed a message requesting them to be more accurate if their error rate exceeded $15 \%$, and to respond more quickly if their error rate was below $5 \%$.

\section{Results}

The mean of subjects' median correct reaction times (RTs), as well as their mean error rates, are shown in Figure 2 for the six conditions. A two-way within-subjects analysis of variance (ANOVA) was conducted on the RT data, to determine the effects of format and string type. It revealed a main effect of format $[F(1,29)=26.4$, $\left.M S_{\mathrm{e}}=5,731, p<.001\right]$, with faster RTs in the tilted-letter conditions. There was also a significant effect of string
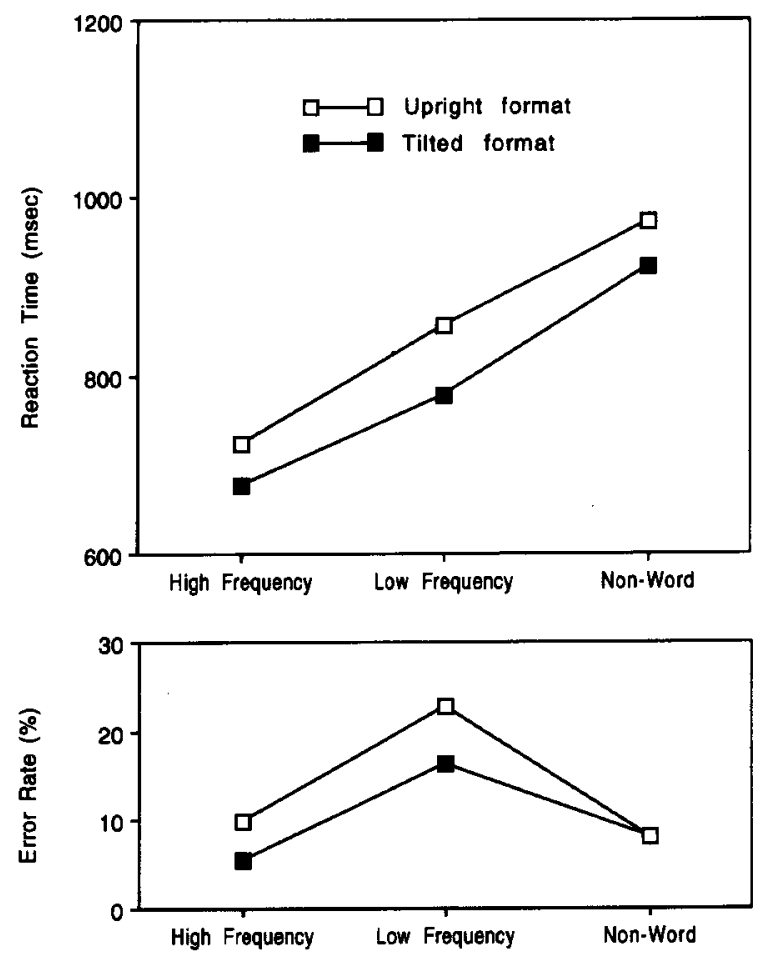

Figure 2. The means of median reaction times (top) and error rates (bottom) to make a correct lexical decision in Experiment 1. Stimuli were either words of high or low frequency or pronounceable nonwords. They were lowercase and presented in columnar format, with the letters either all upright (open squares) or all tilted $90^{\circ}$ (solid squares).

type $\left[F(2,58)=36.4, M S_{\mathrm{e}}=25,337, p<.001\right]$, but no interaction of string type with format $[F(2,58)=2.5$, $\left.M S_{\mathrm{e}}=1,938\right]$. Specific contrasts showed that RTs were slower to nonwords than to low-frequency words $[F(1,29)$ $\left.=16.8, M S_{\mathrm{e}}=124,339, p<.001\right]$, and that they were slower to low-frequency words than to high-frequency words $\left[F(1,29)=83.9, M S_{\mathrm{e}}=19,197, p<.001\right]$.

A similar ANOVA of the error data found a main effect of format $\left[F(1,29)=13.1, M S_{\mathrm{e}}=44.2, p<.001\right]$, with more errors in the upright-letter format. There was also a significant effect of string type $[F(2,58)=39.5$, $\left.M S_{\mathrm{e}}=68.8, p<.001\right]$ and an interaction of string type with format $\left[F(2,58)=11.9, M S_{\mathrm{e}}=13.9\right]$, because format had no effect on nonwords in the error data (see Figure 2). Specific contrasts showed that error rates were higher to low-frequency words than they were either to nonwords $\left[F(1,29)=39.3, M S_{\mathrm{e}}=398.8, p<.001\right]$ or to high-frequency words $\left[F(1,29)=54.5, M S_{\mathrm{e}}=310, p<\right.$ $.001]$. However, there was no difference between error rates to high-frequency words and error rates to nonwords $[F(1,29)=0.2$, n.s. $]$.

\section{Discussion}

These results are consistent with our suggestion, derived from work on nonlinguistic shape perception, that the global configuration of a letter string may provide a 
principal axis or reference frame relative to which its constituent letters are described. Lexical decisions for the present columnar strings were faster when each letter was tilted than when each letter was upright. In the tilted format, the letters had their usual orientation relative to the principal axis of the word, but were misoriented relative to the subject. The reverse applied in the upright format. Thus, one interpretation of the tilted-format advantage would be that the orientation of letters relative to the principal axis of the string is more important than their orientation relative to the subject. This implies letter coding relative to the principal axis of the string.

Koriat and Norman (1985, Experiment 4) previously obtained a similar result (of which we were unaware at the time of running this study), and also provided an alternative explanation. They found an advantage for the tilted format over the upright format for lexical decisions to Hebrew letter strings presented at $30^{\circ}, 70^{\circ}, 290^{\circ}$, and $330^{\circ}$ orientations. The present experiment extends this finding to English text at $90^{\circ}$. In contrast to our axisbased hypothesis, Koriat and Norman (1985) interpreted their findings as evidence for the involvement of "transgraphemic" features in word recognition. Specifically, under the tilted format, the global shape that the string would have under conventional format is preserved; this global shape simply undergoes a $90^{\circ}$ rotation. By contrast, the usual global shape is completely disrupted in the upright format. Several authors have suggested that words may be recognized on the basis of global visual properties, such as their low spatial-frequency envelope (e.g., Crowder, 1982; Garner, 1981; Monke \& Hulme, 1983 ), and it is possible that the current tilted-format advantage arose because only the upright format disrupts these cues. We term this account of the tilted-format advantage the global-shape hypothesis.

There are several reasons to question this account. The existing evidence for word recognition by global envelope is not entirely compelling (see Henderson, 1987). For instance, the word-superiority effect (e.g., Reicher, 1969) was originally attributed to global shape, but has since been obtained under conditions of case alternation (cAsE aLtEr$\mathrm{NaTiOn)}$ that are presumed to disrupt any familiar global envelope (e.g., Adams, 1979; Besner, 1983; McClelland, 1976). Several other effects that were once attributed to global shape are similarly preserved under case alternation (e.g., Besner, Davelaar, Alcott, \& Parry, 1984; Besner, 1989). Nevertheless, we ran a further experiment to test the global-shape account of the tilted-format advantage.

\section{EXPERIMENT 2}

This study was an exact replication of Experiment 1, except that all the letter strings were now presented in alternating case, with the first letter always in lowercase (see Figure 1B for examples). As a result, any familiar global envelope should be disrupted in both formats. If the format effect observed in Experiment 1 was primarily due to preservation of global envelopes under the tilted format only, the effect should be abolished or re- duced in Experiment 2. On the other hand, if the advantage for the tilted format was caused by letter description relative to the principal axis of the string, it should be replicated. Thirty new subjects, from the same source as those used in Experiment 1, participated.

\section{Results}

Figure 3 shows the mean of subjects' median correct RTs, together with their mean error rates, for the six conditions. These results were similar to those obtained in Experiment 1 (compare with Figure 2). A two-way withinsubjects ANOVA was conducted on the RT data, to determine the effects of format and string type. This revealed a main effect of format $\left[F(1,29)=11.7, M S_{\mathrm{e}}=15,220\right.$, $p<.002]$, with faster RTs in the tilted-letter conditions. There was also a significant effect of string type $\left[F(2,58)=9.4, M S_{\mathrm{e}}=191,292, p<.001\right]$, but there was no interaction of string type with format $[F(2,58)=2.5$, $\left.M S_{\mathrm{e}}=1,938\right]$. Specific contrasts showed that RTs were slower to nonwords than they were to low-frequency words $\left[F(1,29)=6.5, M S_{\mathrm{e}}=124,339, p<.02\right]$, and that they were slower to low-frequency words than to high-frequency words $\left[F(1,29)=65.9, M S_{\mathrm{e}}=16,013, p<.001\right]$.

A similar ANOVA of the error data found no effect of format $\left[F(1,29)=2.4, M S_{\mathrm{e}}=19.0\right]$, although error
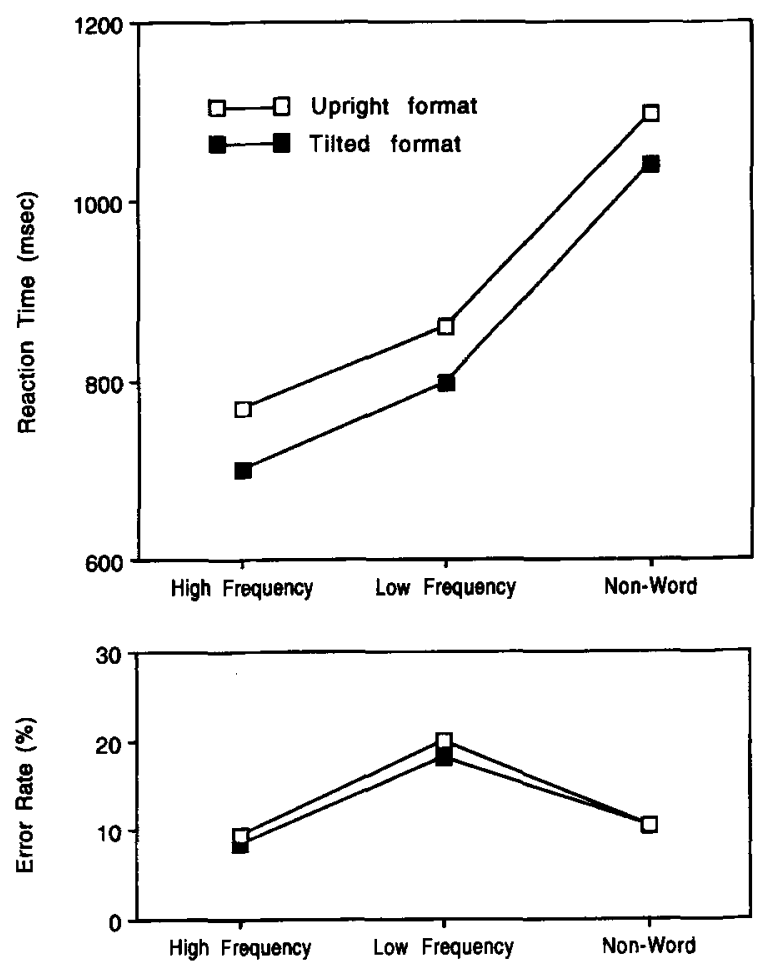

Figure 3. The means of median reaction times (top) and mean error rates (bottom) to make a correct lexical decision in Experiment 2. As in Experiment 1, the stimuli were either high- or lowfrequency words or pronounceable nonwords, presented in columnar format with upright letters (open squares) or tilted letters (solid squares). However, they were now presented in alternating case to disrupt any familiar global shapes. The scale matches Figure 2 to facilitate comparison between the experiments. 
rates were lower in the tilted-letter conditions for both types of words. There was a significant effect of string type $\left[F(2,58)=23.7, M S_{\mathrm{e}}=75.6, p<.001\right]$, but there was no interaction of string type with format $[F(2,58)=$ $\left.0.5, M S_{\mathrm{e}}=23.7\right]$. Specific contrasts showed that error rates were higher to low-frequency words than they were either to nonwords $\left[F(1,29)=20.1, M S_{\mathrm{e}}=444.8, p<\right.$ $.001]$ or to high-frequency words $\left[F(1,29)=63.1, M S_{\mathrm{e}}=\right.$ $194.4, p<.001]$. However, there was no difference between the error rates to high-frequency words and the error rates to nonwords $\left[F(1,29)=1.0, M S_{\mathrm{e}}=267.4\right]$.

\section{Discussion}

Experiment 2, in which all stimuli were presented in alternating case to disrupt any familiar global shape, replicated the main findings of Experiment 1 . The tiltedformat advantage was still observed and cannot, therefore, be attributed to the preservation of familiar (albeit rotated) global envelopes for words in only the tilted conditions. This envelope version of the global-shape hypothesis can therefore be rejected, at least as an account of the present tilted-format advantage. The results remain consistent with our alternative interpretation in terms of reference frames. It appears that the orientation of letters relative to the principal axis of the string can be more important in determining word perception than their orientation relative to the subject. As discussed earlier, this conclusion would accord with models of nonlinguistic shape perception (e.g., Palmer \& Bucher, 1981) in which component shapes (in the present case, letters) are described relative to the principal axis of the global configuration (in this case, the letter string).

Although the effects of word frequency are largely tangential to our main concerns, we may briefly note that they provide another line of evidence against any account of the tilted-format advantage in terms of global envelope. High-frequency words should have the most familiar, and therefore the most useful, global envelopes (see Besner, 1989; Haber \& Haber, 1981). Thus, disrupting the global envelope via the upright columnar format, or via the use of alternating case, should be most detrimental for high-frequency words. No such interaction with frequency was observed in Experiments 1 or 2 . Indeed, in Experiment 1, the means show a greater cost for the upright format with low-frequency words (see Figure 2).

The global-shape hypothesis therefore seems inconsistent with our frequency data. Perhaps one could question the common assumption that global envelope is most useful for high-frequency words, and somehow reconcile the present frequency data with a role for such global shape. However, even this revised global-shape hypothesis would remain inconsistent with the basic finding of Experiment 2, in which the tilted format completely retained its advantage, found in Experiment 1, over the upright, despite the change to alternating case. This implies that global envelope plays no role in the tilted-format advantage.

\section{EXPERIMENT 3}

Our next study examined a further alternative to our axis-based hypothesis as an explanation for the tiltedformat advantage. This effect might conceivably be the trivial outcome of a difference in intraletter spacing between the two formats. We considered this an unlikely explanation, since spacing has little or no effect on lexical decision (beyond acuity factors) for strings in conventional horizontal format, or for strings in various rotated formats (e.g., Koriat \& Norman, 1985). Moreover, in the preceding studies, we had attempted to equate the spacing between individual letters in the two formats by matching their center-to-center spacing. Nevertheless, there were some inevitable spacing differences between formats, since several lowercase letters are more elongated vertically than they are horizontally, which may result in reduced contour-to-contour separation on average for the columnar strings with upright letters compared with those with tilted letters. This possible spacing artifact may also apply to the previous study of Koriat and Norman (1985, Experiment 3), in which an advantage was found for the tilted format in Hebrew letter strings.

To eliminate any role of such spacing differences, our next study used uppercase letters, each defined within a square matrix (see Figure $1 \mathrm{C}$ for examples). As a result, the vertical and horizontal extent of letters both covered the same range, so that tilting the letters should have no effect on their direction of elongation (at least, no effect on average). Thus, contour-to-contour separation between the letters was now matched for the two formats, in addition to center-to-center separation. Note that in addition to controlling for any effects of interletter spacing, the use of uppercase strings also provides a further means of disrupting any familiar global envelope that might be present in tilted lowercase words.

\section{Method}

Subjects. The 14 new subjects were paid volunteers from the subject panel at the University of California, Berkeley.

Apparatus and Materials. The apparatus was a Viglen 3/33 microcomputer with VGA monitor. Stimulus onset and offset occurred within a single frame by changing the palette look-up table. Uppercase Turbo Pascal sans-serif font was now employed for all letters. We adjusted the vertical-height control on the monitor to ensure that the square pixel matrices defining the letters were actually physically square upon the screen.

The materials were 40 four-letter words and 40 pronounceable nonwords derived by rearranging letters within each of the words, all taken from Mason (1978, Experiment 3). Each appeared three times in a total of 240 trials split into six blocks. The stimuli were randomly assigned to condition on each appearance. In all other respects, the method followed that of the previous studies.

\section{Results}

The means of subjects' median RTs are shown in Figure 4 for each condition, together with the associated error rates. A two-way within-subjects ANOVA of the RT data found a main effect of format $\left[F(1,13)=8.1, M S_{\mathrm{e}}=6,605\right.$, $p=.01]$, with faster RTs for tilted strings. This replicates 

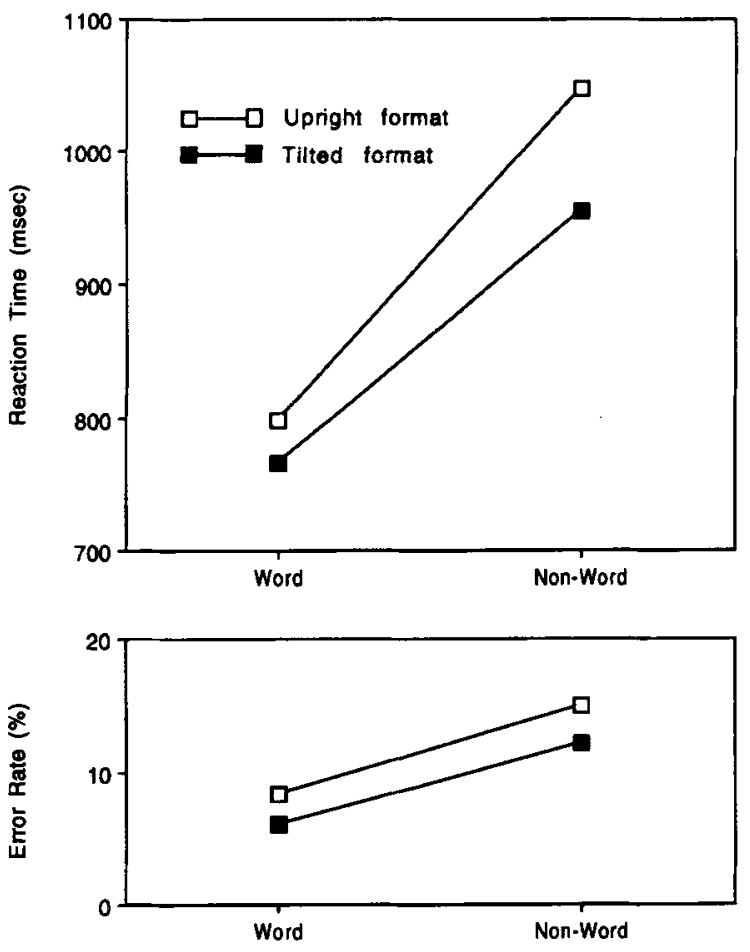

Figure 4. The means of median reaction times (top) and mean error rates (bottom) to make a correct lexical decision in Experiment 3. Stimuli were four-letter words or nonwords, presented in columnar format with upright letters (open squares) or tilted letters (solid squares). Square uppercase letters were used to preclude any spacing differences between the formats.

the tilted-format advantage seen in our previous experiments. There was also an effect of string type $[F(1,13)=$ $\left.12.8, M S_{\mathrm{e}}=52,736, p<.01\right]$, but no interaction $[F(1,13)=$ $2.5, p>.1]$, despite the numerical trend for larger format effects with nonwords. Similarly, analysis of the error data found main effects of format $\left[F(1,13)=10.5, M S_{\mathrm{e}}=8.3\right.$, $p<.01]$ and string type $\left[F(1,13)=12.0, M S_{\mathrm{e}}=46.7, p<\right.$ $.01]$, but no interaction $[F(1,13)=.05$, n.s. $]$.

\section{Discussion}

This experiment replicated the tilted-format advantage seen in our two preceding studies. Since square uppercase letters were employed, the present tilted-format advantage cannot be attributed to any differences in interletter spacing between the two formats. If we assume that presenting the strings entirely in uppercase disrupts global-shape cues that may be present in lowercase words, the present study also agrees with the findings of Experiment 2 (which used alternating case), in showing that the format effect is not caused by the tilted condition preserving any familiar global envelope for words.

\section{EXPERIMENT 4}

Our next study manipulated the number of letters in the columns for both the upright and tilted formats. The speed of lexical decision is scarcely affected by string length for conventional horizontal formats (e.g., Frederiksen \& Kroll, 1976) within the limits of acuity. Indeed, this provides one illustration of the whole being more than the sum of its parts, in the sense that word recognition does not usually proceed in a serial letter-by-letter fashion, but operates instead in a more parallel or configural manner. However, in our account of the present tilted-format advantage, the letters in the upright format are aligned with a vertical axis that is appropriate for individual letter recognition but not for configural recognition. Accordingly, a strategy of letter identification that is more serial than normal may be adopted in the upright format.

Koriat and Norman (1985, Experiments 1, 2, and 3) previously observed that lexical decisions are affected by the number of letters in strings rotated holistically beyond $60^{\circ}$ from the horizontal. On the basis of these prior findings, we would expect some string-length effects in the present tilted format, as well as in the upright format. Our prediction was simply that these effects should be more pronounced in the upright format, given its disadvantage for configural coding. ${ }^{1}$

\section{Method}

Subjects. The 40 new subjects were paid volunteers from the University of Oregon subject panel.

Apparatus and Materials. The apparatus comprised an IBM PS/2 microcomputer with VGA monitor. The 160 stimulus items were taken from Mason (1978, Experiment 3), and comprised 40 fourletter words, 40 six-letter words, and 80 pronounceable nonwords derived by rearranging the letters within each word (the four-letter strings were the same as those used in the current Experiment 3 ). Each string appeared in lowercase letters. There were 480 trials, split into six blocks, with each stimulus appearing once every two blocks, randomly assigned to condition on each appearance, and in a different random order for each subject. In all other respects, the method followed that of Experiments 1, 2, and 3.

The four- and six-letter words in the stimulus set are roughly matched for frequency according to Mason (1978). Nevertheless, we anticipated length effects (i.e., slower RTs for six-letter strings) with the current columnar format, especially when the letters were upright. According to our axis-based hypothesis, the upright letters have the appropriate orientation for individual recognition, but not for conf igural recognition.

Design. A within-subjects design was used, with three factors format (upright vs. tilted letters presented in columns); string type (word vs. nonword); and string length (four vs. six letters). These three factors were crossed to yield eight conditions.

\section{Results}

The means of subjects' median RTs, together with their associated mean error rates, are given in Figure 5 for each condition. A three-way within-subjects ANOVA of the RT data showed a significant effect of format $[F(1,39)=$ $\left.114.5, M S_{\mathrm{e}}=4,065, p<.0001\right]$, with slower RTs for columnar strings that had upright letters. This replicates the basic tilted-format advantage from the previous studies. There was also an effect of string type $[F(1,39)=$ 97.1, $\left.M S_{\mathrm{e}}=10,773, p<.0001\right]$, with slower RTs for nonwords, and an effect of string length $[F(1,39)=97.3$, $\left.M S_{\mathrm{e}}=10,617, p<.0001\right]$, with slower RTs for six-letter 

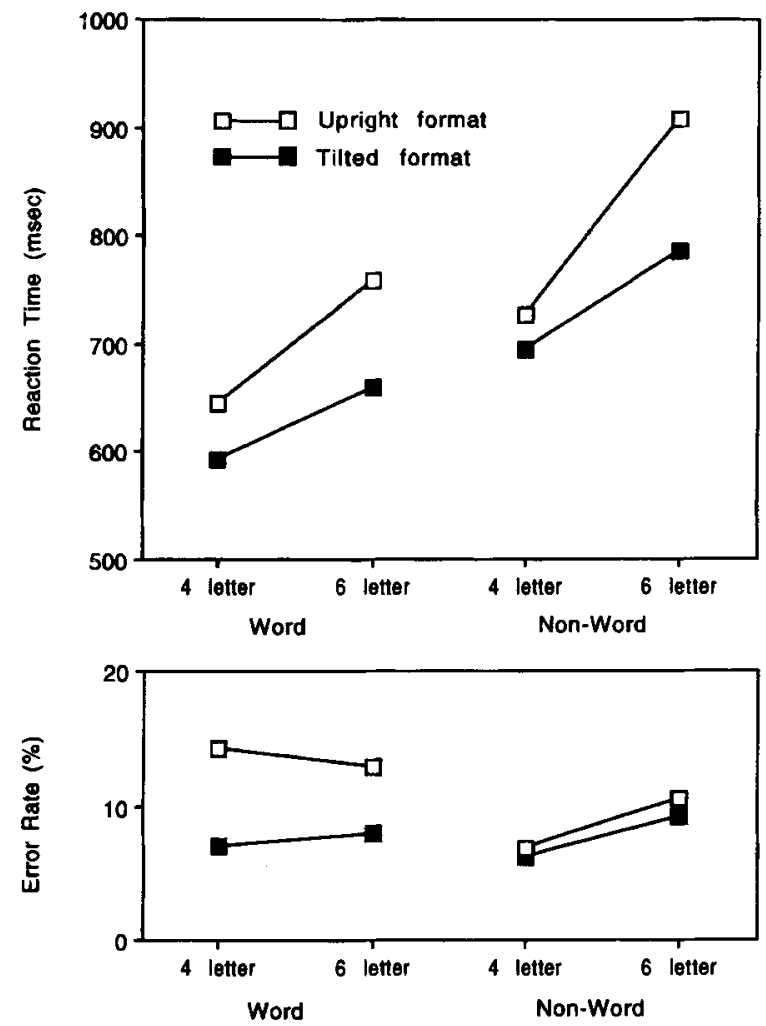

Figure 5. The means of median reaction times (top) and mean error rates (bottom) to make a correct lexical decision in Experiment 4. Stimuli were columnar lowercase letter strings with either upright letters (open squares) or tilted letters (solid squares). They were either words (left lines) or pronounceable nonwords (right lines) with four or six letters.

strings. Format did not interact with string type $[F(1,39)<$ $1]$, but did interact with string length $[F(1,39)=41.6$, $\left.M S_{\mathrm{e}}=2,184, p<.001\right]$. There was a larger cost for sixletter strings than for four-letter strings in the upright format than there was in the tilted format, just as predicted. String length also interacted with string type $\left[F(1,39)=11.1, M S_{\mathrm{e}}=3,530, p<.01\right]$, with larger effects of length for nonwords. Finally, the three-way interaction was just significant $\left[F(1,39)=6.3, M S_{\mathrm{e}}=1,438\right.$, $p<.05]$, because the upright format produced the greatest cost relative to the tilted format in the case of six-letter nonwords.

A similar analysis was carried out on the accuracy data. There were significant effects of format $[F(1,39)=$ 46.6, $\left.M S_{\mathrm{e}}=22.2, p<.001\right]$, string type $[F(1,39)=10.2$, $\left.M S_{\mathrm{e}}=43.0, p<.01\right]$, and string length $[F(1,39)=11.0$, $\left.M S_{\mathrm{e}}=17.0, p<.01\right]$. Format interacted with string type $\left[F(1,39)=15.3, M S_{\mathrm{e}}=32.9, p<.001\right]$, producing a larger effect on words. ${ }^{2}$ Format did not interact with string length $[F(1,39)<1]$. Inspection of Figure 5 suggests a possible speed-error tradeoff in the effect of length for upright words (left-hand lines) but not for nonwords (right-hand lines). However, this error trend for upright words was not consistent across subjects: 18 out of 40 showed more errors with six-letter upright words than they did with four-letter words. Finally, string type showed a significant interaction with string length $\left[F(1,39)=8.1, M S_{\mathrm{e}}=\right.$ $30.2, p<.01]$, with larger effects of length for nonwords (as in the RT data). The three-way interaction was not significant $[F(1,39)=1.5]$.

\section{Discussion}

Lexical decision for letter strings presented in conventional horizontal format (e.g., Frederiksen \& Kroll, 1976) is generally unaffected by the number of letters (although Mason [1978, Experiment 3] found small effects of string length on naming time using the present set of words and nonwords). Using columnar rather than horizontal strings, we found substantial string-length effects on lexical decision in both the tilted and upright formats. More importantly, these length effects were more pronounced for the upright format than for the tilted format. Although this result provides rather indirect evidence for the suggested role of principal axes, it is certainly consistent with our proposal that upright letters in columnar strings are aligned with a vertical principal axis that is appropriate for individual letter recognition, but inappropriate for configural processing.

\section{GENERAL DISCUSSION}

In four experiments, we found that lexical decision to columnar letter strings was easier when each letter was tilted $90^{\circ}$ (as if the entire string were rotated $90^{\circ}$ from conventional format) than it was when each letter remained upright (see Figure 1 for examples of these two formats). An advantage for the tilted format has been observed before in experiments that focused on other issues (Koriat \& Norman, 1985, Experiment 4, with Hebrew strings rotated $30^{\circ}$ or $70^{\circ}$ from the horizontal). These previous findings simply underline the robustness of the phenomenon under investigation here. As a further informal demonstration of the effect, we may note that the many publishers who print titles along book spines using tilted letters cannot all be wrong! In the present studies, we examined the boundary conditions for this tilted-format advantage, and assessed its significance.

In Experiment 2, we found that the tilted-format advantage was fully preserved when lowercase strings were replaced with alternating case to remove any familiar global envelope that might otherwise be preserved for words in the tilted condition, but disrupted in the upright condition. Similarly, in Experiment 3 we found a tiltedformat advantage when square uppercase letters were used throughout each string. This manipulation was also intended to disrupt any familiar global shapes, in addition to ensuring comparable spacing for the two formats. Finally, in Experiment 4 the advantage of the tilted format over the upright format increased with the number of letters in the string. In the light of these new findings, what account can be offered for the tilted-format advantage, and what are its possible implications for normal reading?

The account we developed in our introduction was based upon observations that shape perception in nonlinguistic 
domains can be strongly influenced by reference frames in the form of principal axes (e.g., Humphreys, 1983). In particular, axes that are determined by global configurations can influence the perception of local shapes within the configuration (e.g., Palmer 1985; Palmer \& Bucher, 1981). We suggested that similar principles may apply to word perception by the visual system. Thus, the description of local components (in this case, letters) may take place relative to a reference frame or principal axis determined by the global configuration (i.e., the whole letter string). This possibility has been raised before in the context of connectionist models of visual word recognition. For instance, Hinton and Shallice's (1991, p. 78) connectionist system assumes an input of letters "represented relative to a reference frame based on the word itself" (our emphasis).

The tilted-format advantage we have found is consistent with this characterization of word recognition in terms of intrinsic reference frames. In the tilted condition, the letters are misoriented relative to the subject, but have their usual orientation relative to the principal axis of the string. In the upright condition, the reverse applies - that is, the letters are normally oriented relative to the subject, but misoriented relative to the principal axis. The fact that the tilted strings are easier to judge suggests that the reference frame provided by the string is more important than any egocentric frames provided by the subject.

\section{The Possible Role of Mental Rotation}

One might suggest that the tilted-format advantage arises not because of axis-based coding, but because the tilted format can be transformed to the conventional row format by a $90^{\circ}$ mental rotation (Cooper \& Shepard, 1973), while the upright format would remain unfamiliar even after such a transformation. Below, we argue, first, that it remains doubtful whether such rotation is required when reading columnar strings, and second, that any tenable account involving rotation reduces in any case to the main conclusions of our axis-based account.

While the most substantial length effect in the upright format was found in Experiment 4, the tilted format was also affected by the number of letters in the string. This rules out a holistic rotation process applied to the entire tilted string as a single object (although piecemeal rotation does remain a possibility). Our length effect in the tilted strings is corroborated by the more extensive observations of Koriat and Norman (1985), who systematically examined the possibility that subjects read misoriented Hebrew letter strings by means of mental rotation. They presented strings only in our tilted format, at a large range of orientations. Their results showed that for angular deviations of less than $60^{\circ}$ from the horizontal, neither orientation nor string length had any effect on lexical decision. Strings within this range around the horizontal are apparently processed just like horizontal strings. For orientations beyond $60^{\circ}$ (and spanning the $90^{\circ}$ orientation employed here), nonlinear effects of orientation were found, and these increased sharply with string length, arguing against any holistic rotation pro- cess. Koriat and Norman (p. 504) concluded that the case for mental rotation during reading remains unproven, cautioning that "it is not clear whether the reading of rotated words involves the same sort of mental rotation process envisioned by Shepard and colleagues."

Suppose some form of mental rotation (presumably piecemeal, given the length effects) actually did occur: Could such rotation explain our findings? Given our findings with alternating case (Experiment 2) and uppercase (Experiment 3 ) strings, it is clear that the tiltedformat advantage cannot be due to familiarity with any global envelope produced following rotation. The only remaining difference in form between rotated representations of the tilted format and those for the upright format would be that only in the tilted case do the letters have their usual relation to the string's principal axis. Thus, on closer inspection, the issue of whether mental rotation takes place becomes moot. Even if the tilted strings are mentally rotated, their advantage must lie in the relation of their component letters to the principal axis of the string.

\section{Axis-Based Coding of Letter Shape Versus Axis-Based Coding of Letter Position}

We derived our axis-based account from theories of nonlinguistic shape perception that propose that local shapes are described relative to axes determined by the global configuration (e.g., Palmer \& Bucher, 1981). Applying these notions to the current results implies that the tilted-format advantage results from enhanced shape perception for the letters when they have their usual relation to the principal axis of the string. However, work by Koriat and Norman (1989) suggests that such emphasis on shape coding within misoriented letter strings may be inappropriate.

Koriat and Norman (1989) were intrigued by the apparent paradox that whereas word recognition is substantially impaired by misorientation (beyond about $60^{\circ}$, as noted above), the identification of single letters is rarely affected at all by misorientation. They suggested that this arises because misorientation critically impairs perception of the relative position of letters within strings, not perception of their shape. In support of this configural position-coding account, Koriat and Norman found strong effects of misorientation whenever the task required discrimination of letter position within the string (as for the lexical decision tasks used here), but not when position was irrelevant to the task (as in the identification of single letters).

It is possible that the present tilted-format advantage reflects a benefit for within-string letter-position coding when each letter is aligned with the principal axis, rather than any benefit in shape perception. In other words, the disruption of position coding that Koriat and Norman (1989) observed for the tilted format may be even more pronounced with our upright format. Further research is required to distinguish the effect of axis alignment on shape coding for letters from its effect on the coding of their position in the string. Our tilted and upright formats 
should be compared in tasks that only require the identity of the letters, as well as in tasks that require information about the relative position of the letters in each string (such as the present lexical decision task).

To conclude, in four experiments, we found that lexical decision to columnar letter strings was easier when all the letters were tilted $90^{\circ}$ than it was when all the letters were upright. These results cannot be attributed to the preservation of familiar global envelopes in the former case. They suggest a role for the principal axis of letter strings in providing a reference frame relative to which the component letters are described. This general conclusion is consistent with models of nonlinguistic shape perception. However, there are at least two ways in which the reference frame provided by the string's principal axis may play a role: First, by influencing shape perception for the individual letters; and second, by influencing the coding of letter position within a string. We are currently designing experiments to distinguish these possibilities.

\section{REFERENCES}

Adams, M. J. (1979). Models of word recognition. Cognitive Psychology, 11, 133-176.

ATtNEAVE, F. (1968). Triangles as ambiguous figures. American Journal of Psychology, 81, 447-453.

BESNER, D. (1983). Basic decoding components in reading: Two dissociable feature extraction processes. Canadian Journal of Psychology, 37, 429-438.

BESNER, D. (1989). On the role of outline shape and word specific visual pattern in the identification of function words: NONE. Quarterly Journal of Experimental Psychology, 41A, 91-105.

Besner, D., DavelaAr, E., AlcotT, D., \& Parry, P. (1984). Wholistic reading of alphabetic print: Evidence from the FDM and the FBI. In L. Henderson (Ed.), Orthographies and reading (pp. 121-135). London: Erlbaum.

BESNER, D., \& MCCANN, R. S. (1987). Word frequency and pattern distortion in visual word identification and production: An examination of four classes of models. In M. Coltheart (Ed.), Attention and performance XII (pp. 201-219). Hillsdale, NJ: Erlbaum.

COOPER, L. A., \& ShEPARD, R. N. (1973). Chronometric studies of the rotation of mental images. In W. G. Chase (Ed.), Visual information processing (pp. 75-146). New York: Academic Press.

Corballis, M. C., \& NaGourneY, B. A. (1978). Latency to categorize disorientated alphanumeric characters as letters or digits. Canadian Journal of Psychology, 32, 186-188.

Corballis, M. C., Zbrodoff, N. J., Shetzer, L. I., \& Butler, P. B. (1978). Decisions about identity and orientation of rotated letters and digits. Memory \& Cognition, 6, 98-107.

CROWDER, R. G. (1982). The psychology of reading. Oxford: Oxford University Press.

ELEY, M. G. (1982). Identifying rotated letter-like symbols. Memory \& Cognition, 10, 25-32.

Frederiksen, J. R., \& Krolx, J. F. (1976). Spelling and sound: Approaches to the internal lexicon. Journal of Experimental Psychology: Human Perception \& Performance, 2, 361-379.

GARNER, W. R. (1981). The role of configuration in the identification of visually degraded words. Memory \& Cognition, 9, 445-452.

HABER, R. N., \& HABER, L. R. (1981). Visual components of the reading process. Visible Language, 15, 147-182.

HENDERSON, L. (1987). Word recognition: A tutorial review. In M. Coltheart (Ed.), Attention and performance XII (pp. 171-200). Hillsdale, NJ: Erlbaum.
Hinton, G. E., \& Shallice, T. (1991). Lesioning an attractor network: Investigations of acquired dyslexia. Psychological Review, 98, 74-95.

HUEY, E. B. (1968). The psychology and pedagogy of reading. Cambridge, MA: MIT Press. (Original work published 1908)

HuMPHREYs, G. W. (1983). Reference frames and shape perception. Cognitive Psychology, 15, 151-196.

Humphreys, G. W., \& Quinlan, P. T. (1988). Priming effects between two-dimensional shapes. Journal of Experimental Psychology: Human Perception \& Performance, 14, 203-220.

JoliCOEUR, P., \& LANDAU, M. J. (1984). Effects of orientation on the identification of simple visual patterns. Canadian Journal of Psychology, 38, 80-93.

KorIat, A., \& Norman, J. (1985). Reading rotated words. Journal of Experimental Psychology: Human Perception \& Performance, 11, 490-508.

Koriat, A., \& Norman, J. (1989). Why is word recognition impaired by disorientation while the identification of single letters is not? Journal of Experimental Psychology: Human Perception \& Performance, 15, 153-163.

MACH, E. (1959). The analysis of sensations (C. M. Williams, Trans.). New York: Dover. (Original work published 1914)

MARR, D., \& NishiHARA, H. K. (1978). Representation and recognition of the spatial organisation of three-dimensional shapes. Proceedings of the Royal Society of London: Series B, 200, 269-294.

MASON, M. (1978). From print to sound in mature readers as a function of reader ability and two forms of orthographic regularity. Memory \& Cognition, 6, 568-581.

MCClelland, J. L. (1976). Preliminary letter recognition in the perception of words and nonwords. Journal of Experimental Psychology: Human Perception \& Performance, 2, 80-91.

McClelland, J. L., \& RumelharT, D. E. (1981). An interactive activation model of context effects in letter perception: 1 . An account of basic findings. Psychological Review, 88, 375-407.

MonK, A. F., \& Hulme, C. (1983). Errors in proofreading: Evidence for the use of word shape in word recognition. Memory \& Cognition, 11, 16-23.

PALMER, S. E. (1980). What makes triangles point: Local and global effects in configurations of ambiguous triangles. Cognitive Psychology, 12, 285-305.

PALMER, S. E. (1985). The role of symmetry in shape perception. Acta Psychologica, 59, 67-90.

PALMer, S. E., \& BuCher, N. M. (1981). Configural effects in perceived pointing of ambiguous triangles. Journal of Experimental Psychology: Human Perception \& Performance, 7, 88-114

Reicher, G. M. (1969). Perceptual recognition as a function of meaningfulness of stimulus material. Journal of Experimental Psychology, 81, 274-280.

Rock, I. (1973). Orientation and form. New York: Academic Press. WhiTe, M. J. (1980). Naming and categorization of tilted alphanumeric characters do not require mental rotation. Bulletin of the Psychonomic Society, 15, 153-156.

\section{NOTES}

1. By "configural coding," we do not refer to recognition by global envelope, since this possibility was discounted by Experiments 2 and 3. The possible forms that configural coding may take are considered in the General Discussion section.

2. This interaction between format and word versus nonword replicates the pattern of errors from Experiments 1 and 2. One account of the interaction would be that since columns of upright letters are difficult to recognize as words (as shown by the cost relative to tilted letters in each experiment), the upright format produces a bias toward the nonword response, and this artifactually improves accuracy for upright nonwords.

(Manuscript received June 16, 1993; revision accepted for publication July 7,1994 .) 